\title{
Steady-state analysis of a asymmetric bistable system driven by noises with periodic signal
}

\author{
FU Yan \\ Department of General Studies, Nanchang Institute of Science \& Technology, Nanchang, \\ 330108, China \\ E-mail:501284253@qq.com
}

Keywords: noise; asymmetric bistable system; stationary probability distribution; Langevin equation

Abstract. The steady-state properties distribution (SPD) is investigated in a asymmetric bistable system driven by cross-correlated multiplicative white and additive white noises with periodic signal.Using the Liouville Equation and Novikov Theorem,the approximate Fokker-Planck equation (AFPE) is derived,and the analytic expressions of the stationary probability is obtained.

\section{Introduction}

Dynamical properties of a bistable system[1] with noises is a very typical and important problem in statistical mechanics[2].The investigation of dynamical system with correlation noises has attracted a lot attention in the field of stochastic recently[3-12].Correlated noises have been applied to many fields,such as laser system[3],biology system[4] and bistable system[5],and so on.In many cases,noises play a positive role,and many interesting results are found. Fulinski and Telejko[6] have investigated the bistable system driven by correlated additive and multiplicative white noises,and they pointed out that the correlation of the noises changes the dynamics of the system.Zhang Jing-jing[7] and Jin Yan-fei have investigated the mean first-passage time (MFPT) and stochastic resonance (SR) in an asymmetric bistable system driven by multiplicative non-Gaussian noise and additive Gaussian white noise by using the path integral approach and two-state theory. Miao Zu-yingand Wang Hua have studied the SR phenomenon in a bistable system under the simultaneous action of multiplicative and additive noise and periodic signal by using the theory of signal-to-noise ratio (SNR) in the adiabatic limit[8-12].

The largest amount of work mentioned above has been referred to consideration of the symmetric potential. However, symmetry is difficult to guaranteed, and more realistic models require considering asymmetric potential. The effects of asymmetry on the bistable system are gaining more and more attention. In addition, articles on the studies about steady-state analysis in a asymmetric system driven by cross-correlated multiplicative white and additive white noises with periodic signal are few.

This paper has used the Liouville Equation and Novikov Theorem to obtain the analytical expression of the SPD.The effects of the noise parameters and the periodic signal on the SPD of the system are discussed separately, and found some new nonlinear phenomena.

\section{The steady-state probability distribution function}

Consider a conventional one-dimensional asymmetric bistable system driven by cross- correlated multiplicative white and additive white noises with periodic signal[13].Its Langevin equation (LE)[14] reads

$$
\frac{d x}{d t}=-r+x-x^{3}+x \xi(t)+\eta(t)+A \cos \Omega t
$$

where $\xi(t)$ and $\eta(t)$ are the Gaussian white noises with zero-mean.The two noise terms are characterized by their means and variances,and the statistical properties of the noise terms are characterized by their first and second moments[15]: 


$$
\begin{aligned}
<\xi(t)>=<\eta(t)>=0(2) \quad\left\langle\xi(t) \xi\left(t^{\prime}\right)>=2 D \delta\left(t-t^{\prime}\right)(3) \quad<\eta(t) \eta\left(t^{\prime}\right)>=2 Q \delta\left(t-t^{\prime}\right)(4)\right. \\
<\xi(t) \eta\left(t^{\prime}\right)>=<\eta(t) \xi\left(t^{\prime}\right)>=2 \lambda \sqrt{D Q} \delta\left(t-t^{\prime}\right)(5)
\end{aligned}
$$

Where $D$ and $Q$ are the multiplicative and additive noise intensities,respectively.The parameter $\lambda$ measures the strength of the correlations between multiplicative noise $\xi(t)$ and additive noise $\eta(t), r$ is the asymmetric parameter. A denotes the amplitude of the periodic signal, $\Omega$ is the frequency of the periodic signal, and tenotes the time.

To obtain the FPE from the LE (1) with Eq. (2)-(5), Eq. (1) with Eq. (2)-(5) can be transformed into a stochastic equivalent Stratonovich stochastic differential equation

$$
\frac{d x}{d t}=f(x)+g_{1}(x) \xi(t)+g_{2}(x) \eta(t)+A \cos \Omega t
$$

In order to obtain the SPD for Eq. (1), we compare Eq. (1) with Eq. (6) and get

$$
f(x)=-r+x-x^{3}+A \cos \Omega t \quad(7) \quad g_{1}(x)=x \quad \text { (8) } \quad g_{2}(x)=1 \quad \text { (9) }
$$

According to the Liouville Equation, the FPE corresponding to the LE (1) can be written as

$$
\frac{\partial P(x, t)}{\partial t}=-\frac{\partial}{\partial x}\left[f(x)+g_{1}(x) \xi(t)+g_{2}(x) \eta(t)\right] P(x, t)
$$

here we have considered an ensemble of systems in space obeying Eq. (1) for a given realization of $\xi(t)$ and $\eta(t)$ and different initial conditions. It is well known that $\rho(x, t)$ is just the average of $\delta(x(t)-x)$ over the initial conditions, where $x(t)$ is a solution of Eq. (1) for a given realization of $\xi(t)$ and $\eta(t)$ and for a given initial condition, while $x$ is a point in state space. Because the probability density $P(x, t)$ is obtained by averaging $\rho(x, t)$ over the realizations of $\xi(t)$ and $\eta(t)$ (this is known as van Kampen's lemma), we have

$$
\rho(x, t)=<P(x, t)>=<\delta(x-t)>
$$

Here the average $<>$ is taken over initial conditions and over the realizations of $\xi(t)$ and $\eta(t)$. So that the evolution equation for the probability density $P(x, t)$ is average Eq. (10):

$$
\frac{\partial \rho(x, t)}{\partial t}=-\frac{\partial}{\partial x} f(x) \rho(x, t)-\frac{\partial}{\partial x} g_{1}(x)<\xi(t) \delta(x(t)-x)>-\frac{\partial}{\partial x} g_{2}(x)<\eta(t) \delta(x(t)-x)>
$$

The average which remains in Eq. (12) may be calculated for Gaussian noises $\xi(t)$ and $\eta(t)$ by a functional formula, the Novikov theorem:

$$
<\zeta_{k} \Phi\left[\zeta_{1}, \zeta_{2}\right]>=\int_{0}^{t} d t^{\prime} \gamma_{k d}\left(t, t^{\prime}\right)<\frac{\delta(\delta(x(t)-x))}{\delta \zeta_{l}\left(t^{\prime}\right)}>,(k, l=1,2)
$$

According to the Novikov theorem and Fox approach, the AFPE corresponding to Eq.(1) with Eq. (2)-(5) is obtained as follows:

$$
\begin{aligned}
& \frac{\partial P(x, t)}{\partial t}=-\frac{\partial}{\partial x}\left\{f(x)+D g_{1}(x) g_{1}^{\prime}(x)+\lambda \sqrt{D Q}\left[g_{1}(x) g_{2}^{\prime}(x)+g_{1}^{\prime}(x) g_{2}(x)\right]+Q g_{2}(x) g_{2}^{\prime}(x)\right\} P(x, t) \\
& +\frac{\partial^{2}}{\partial x^{2}}\left[D g_{1}^{2}(x)+2 \lambda \sqrt{D Q} g_{1}(x) g_{2}(x)+Q g_{2}^{2}(x)\right] P(x, t)
\end{aligned}
$$

Rewrite Eq. (14) in a more general form as

$$
\frac{\partial P(x, t)}{\partial t}=-\frac{\partial}{\partial x} A(x) P(x, t)+\frac{\partial^{2}}{\partial x^{2}} B(x) P(x, t)
$$

where

$$
A(x)=f(x)+D g_{1}(x) g_{1}^{\prime}(x)+\lambda \sqrt{D Q}\left[g_{1}(x) g_{2}^{\prime}(x)+g_{1}^{\prime}(x) g_{2}(x)\right]+Q g_{2}(x) g_{2}^{\prime}(x)
$$

and

$$
B(x)=D g_{1}^{2}(x)+2 \lambda \sqrt{D Q} g_{1}(x) g_{2}(x)+Q g_{2}^{2}(x)
$$

Solving Eq. (15) for $P(x, t)$ gives us

$$
P_{s t}(x)=\frac{N}{\sqrt{B(x)}} \exp \left(\int^{x} \frac{f(x)}{B(x)} d x\right)
$$

After integrating Eq. (18), we have 


$$
P_{s t}(x)=\frac{N}{\sqrt{B(x)}} \exp \left[-\frac{x^{2}}{2 D}+\frac{2 \lambda \sqrt{D Q}}{D^{2}} x+\frac{2 \lambda \sqrt{D Q}}{D^{2}} A_{0} \frac{1}{2 D} \ln B(x)+\frac{2 \lambda \sqrt{D Q}}{D^{2}} A_{0} \frac{1}{2 D} \ln B(x) \cdot \frac{C_{0}}{2 D^{2}} \frac{1}{\sqrt{\frac{Q}{D}\left(1-\lambda^{2}\right)}} \arctan \frac{x+\lambda \sqrt{\frac{Q}{D}}}{\sqrt{\frac{Q}{D}\left(1-\lambda^{2}\right)}}\right]
$$

where

$$
A_{0}=\frac{D(D+Q)}{2 \lambda \sqrt{D Q}}-2 \lambda \sqrt{D Q} \quad(20) \quad B_{0}=\frac{D^{2} A \cos \Omega t}{2 \lambda \sqrt{D Q}}-\frac{D^{2} r}{2 \lambda \sqrt{D Q}}-Q \quad \text { (21) } \quad C_{0}=\frac{2 D B_{0}}{A_{0}}-2 \lambda \sqrt{D Q}
$$

\section{Discussion}

Making using of the expression Eq.(19) of the $P(x, t)$, the steady-state properties in the asymmetric bistable system can be analyzed by the numerical calculation.
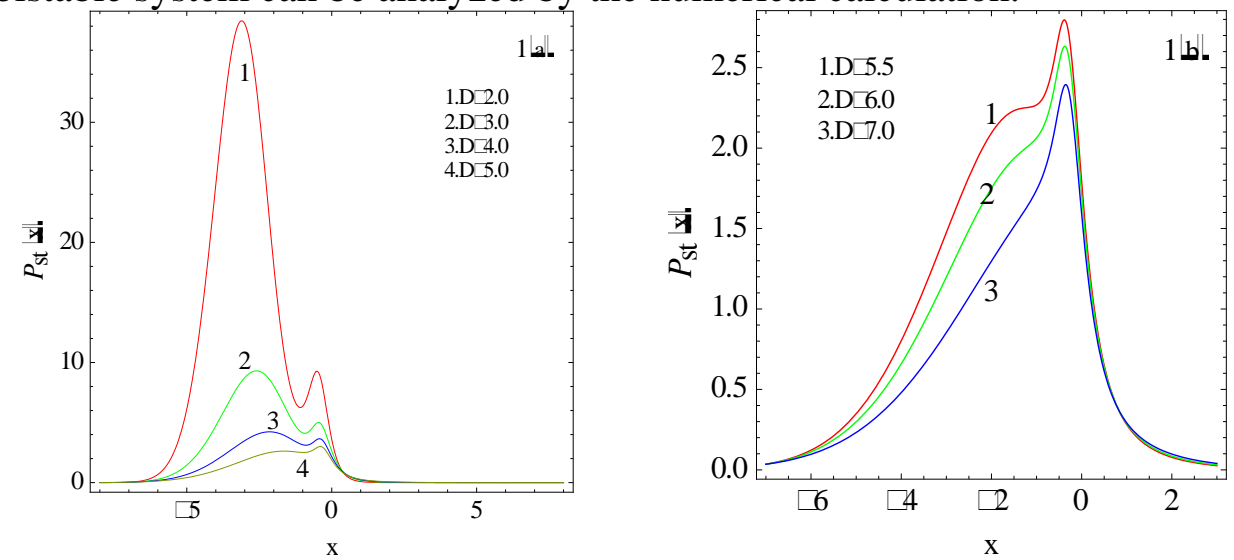

Fig.1 (a) $P(x, t)$ as a function of $x$ with $r=10, \lambda=0.7, Q=2 \quad A=0.5, \Omega=1, t=10$ and $D$ takes 2,3 and 4 (b) $P(x, t)$ as a function of $x$ with $r=10, \lambda=0.7, Q=2, A=0.5, \Omega=1, t=10$ and $D$ takes 5.5, 6 and 7

The result of the numerical calculation of the $P(x, t)$ as a function of $x$ for different values of the $D$ is plotted in Fig.1 (a) and Fig.1 (b), respectively. Figure 1 (a) shows the case of bimodal and Figure 1(b) shows the case of unimodal. Comparing the two charts, it is obvious that the steady-state properties distribution experiences the transition from bimodal to unimodal in structure when the value of the multiplicative noise intensity is more than 5.5. In addition, Figure 1 (a) shows that the peaks of the steady-state properties distribution drop in height with $D$ increasing and the positions of the maximum of the steady-state properties distribution shift from left to right. Figure 1 (b) shows that the peak of the steady-state properties distribution drop in height with $D$ increasing and the position of the maximum of the steady-state properties distribution do not change.

The steady-state properties distribution as a function of $x$ are plotted in Fig.2 (a) and Fig.2 (b). Figure 2 (a) shows the case of bimodal and Figure 2 (b) shows the case of unimodal. Comparing the two chart, it is obvious that the steady-state properties distribution experiences the transition from bimodal to unimodal in structure when the value of the additive noise intensity is more than 3 . In addition, Figure 2 (a) shows that the peaks of the steady-state properties distribution drop in height with $Q$ increasing and the positions of the maximum of the steady-state properties distribution shift from left to right. Figure 2 (b) shows that the peak of the steady-state properties distribution drop in height with $Q$ increasing and the position of the maximum of the steady-state properties distribution do not change. 


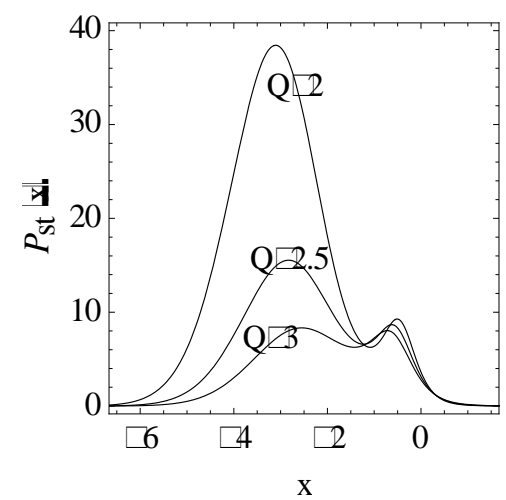

(a)

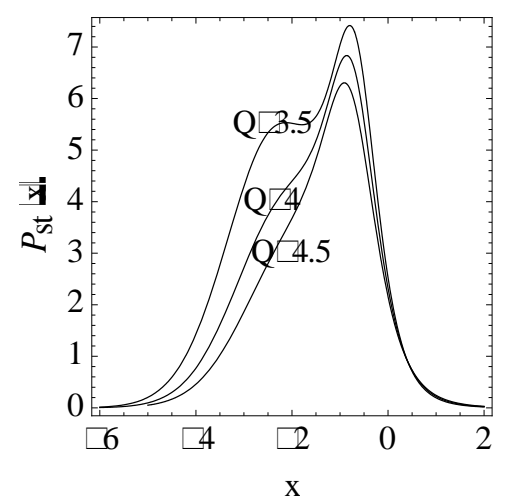

(b)

Fig.2 (a) $P(x, t)$ as a function of $x$ with $r=10, \lambda=0.7, D=2, A=0.5, \Omega=1, t=10$ and Q takes 2,2.5 and 3 (b) $P(x, t)$ as a function of $x$ with $r=10, \lambda=0.7, D=2, A=0.5, \Omega=1, t=10$ and Q takes 3.5,4 and 4.5

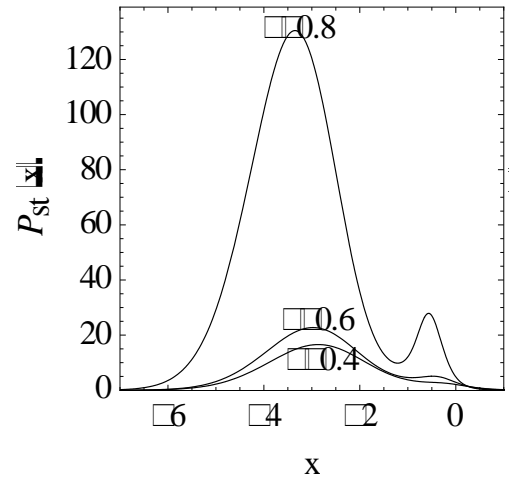

(a)

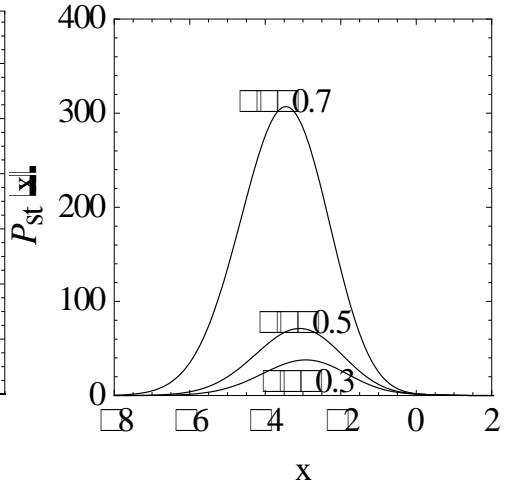

(b)

Fig.3 (a) $P(x, t)$ as a function of $x$ with $r=10, D=2, Q=2, A=0.5, \Omega=1, t=10$ and $\lambda$ takes $0.4,0.6$ and 0.8

(b) $P(x, t)$ as a function of $x$ with $r=10, D=2, Q=2, A=0.5, \Omega=1, t=10$ and $\lambda$ takes $-0.3,-0.5$ and -0.7

The steady-state properties distribution as a function of $x$ are plotted in Fig.3 (a) and Fig.3 (b). Figure 3 (a) shows the case of bimodal and Figure 3 (b) shows the case of unimodal. Comparing the two chart, it is obvious that the steady-state properties distribution experiences the transition from bimodal to unimodal in structure when the value of the cross-correlated intensity is more than 0.4. In addition, Figure 3 (a) shows that the peaks of the steady-state properties distribution drop in height with $\lambda$ increasing and the positions of the maximum of the steady-state properties distribution shift from left to right. Figure 3 (b) shows that the peak of the steady-state properties distribution drop in height with $\lambda$ increasing and the position of the maximum of the steady-state properties distribution shift from left to right.

\section{Conclusions}

In this paper,the relationship between the $P(x, t)$ of the asymmetric bistable system and the variables are analyzed, and some new nonlinear phenomena are obtained, such as:

(1)The multiplicative noise intensity , the additive noise intensity , the cross-correlated intensity between $\xi(t)$ and $\eta(t)$,induce the steady-state properties distribution transition from bimodal to unimodal in structure.

(2) The multiplicative noise intensity, the additive noise intensity, the cross-correlated intensity between $\xi(t)$ and $\eta(t)$ change the peak height of the steady-state properties distribution.

(3) The multiplicative noise intensity, the additive noise intensity , the cross-correlated intensity between $\xi(t)$ and $\eta(t)$ change the positions of the maximum and the minimum of the steady-state properties distribution.

\section{Acknowledgements}


This work is supported by NSFC(Grant Nos.61462032) ,the Science and technology research project of Jiangxi Provincial Department (Grant Nos. GJJ161227), the Science and technology research project of Nanchang Institute of Science and Technology (Grant Nos. NGKJ-17-05, GJKJ-16-01).

\section{References}

[1] Y. Jia, J. R. Li.:Phys.Rev.E 53,5786(1996)

[2] I.S. Gardshteyn, I.M. Ryzhik:Table of Integrals, Series and Products (1980)

[3] S Zhu.:Phys. Rev. A,47 2405(1993)

[4] D C Mei, C.W. Xie and L.Zhang:Eur. Phys. J.B,41 107(2004)

[5] X Q Luo and S Q Zhu:Phys. Rev. E,67 021104 (2003)

[6] Fulnski A,Telejko T. Phys. Lett. A,152 11 (1991)

[7] J J Zhang, Y F Jin. Acta Phys. Sin. 60 12(2011)

[8] Z.D. Yu, C.W. Xie, Z.B. Zhao and C.H. Zeng:Journal of Yunnan University,31 5(2009)

[9] Y.F. Jin, W. Xu, W. Li and S.J. Ma:Journal of Dynamical and Control,3 2(2005)

[10] B.C. Zhou and W. Xu:Acta Phys. Sin. 57 4( 2008)

[11] C.J. Wang , Z.C. Dai and D.C. Mei:Commun.Theor. Phys. 566 (2011)

[12] Z.Y. Miao, H. Wang and S.N. Yu:Journal of Central China Normal University 34 4(2000)

[13] D.J. Wu, L. Cao and S.Z. Ke:Phys. Rev. E50 2496(1994)

[14] B.C. Zhou,W. Xu and X.Y. Zhang:Chinese Journal of Applied Mechanics,26 1 (2009)

[15] C.W. Gardiner:Handbook of Stochastic Methods for Physics,Chemistry and the Natural Sciences, Springer-Verlag(1985) 